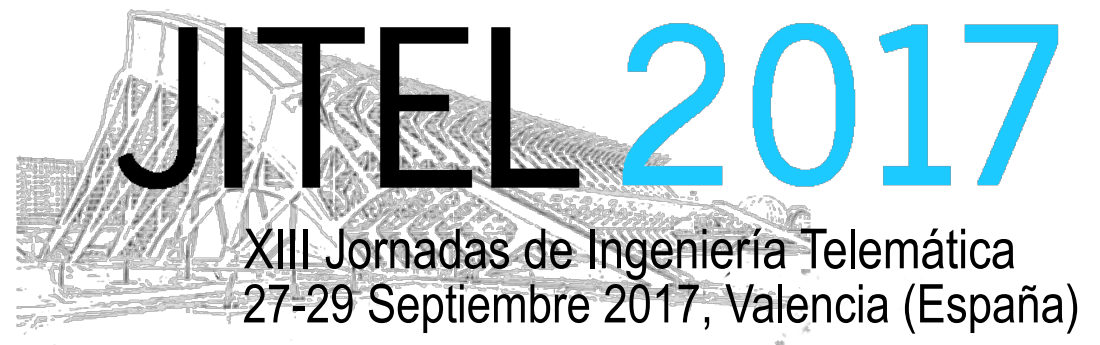

Actas de las XIII Jornadas de Ingeniería

Telemática (JITEL 2017), Valencia (España), 27-29 de

Septiembre de 2017

\title{
QoE en el contexto de Internet of Everything
}

\author{
Maria-Dolores Cano \\ Dpto. Tecnologías de la Información y las Comunicaciones \\ ${ }^{1}$ Universidad Politécnica de Cartagena \\ Campus Muralla del Mar, Edif. Antigones, s/n 30202 Cartagena (España) \\ mdolores.cano@upct.es
}

\begin{abstract}
Resumen- La investigación y el desarrollo hacia la Internet of Everything (IoE) inteligente es un empeño ambicioso $y$ altamente interdisciplinar que debe abordarse en los diferentes niveles de su arquitectura. Un efecto colateral de esta separación se produce en la medición de la calidad de los servicios proporcionados y en cómo evaluar sus prestaciones. La calidad de experiencia de usuario (Quality of user Experience, QoE) se ha convertido en un marco de referencia de evaluación de prestaciones capaz de acoger los nuevos requisitos y demandas de este nuevo paradigma. En este artículo, proporcionamos una visión actual de la QoE en la IoE. Para ello, realizamos un estudio de las propuestas de medición de QoE, de las métricas que se sugieren para su modelado y sus relaciones. Como resultado, se identifica la necesidad de un nuevo enfoque para la evaluación de prestaciones de los servicios y aplicaciones de la IoE capaz de capturar su idiosincrasia, es decir, las nuevas métricas que definen calidad, nivel de conocimiento, nivel de inteligencia, consumo, etc., así como la carencia de un modelado entre los diferentes componentes de la QoE en IoE.
\end{abstract}

Palabras Clave- IoE, IoT, QoE, QoD, QoI.

\section{INTRODUCCIÓN}

Nos encontramos en una situación única en la que nuestro universo físico está adquiriendo una nueva existencia digital, donde cualquier ser/objeto va a estar conectado y capacitado para comunicarse y colaborar. En esta nueva realidad, con millones de dispositivos conectándose a la nube para intercambiar, procesar, y almacenar información, paradigma de Internet of Everything (IoE) [1], la arquitectura de red debe adaptar de forma cognitiva tanto sus principales parámetros de capacidad/caudal y latencia, como la calidad de sus servicios, de la forma más ágil, inteligente y eficiente posible. Es decir, como si se tratase de un servicio público básico (utility service) en función de las demandas de sus productores/consumidores de información.

En este marco general, el proyecto AIM (Augmenting Intelligence, cognitive capabilities, efficiency and value of coMmunication technologies for the $I o E$ ) pretende avanzar en las nuevas tecnologías de red para que permitan crear valor y cubrir efectivamente las necesidades futuras de comunicación y colaboración de una sociedad evolucionada. Durante la última década, se ha realizado un esfuerzo de investigación importante en el ámbito de Internet of Things (IoT), en particular en el avance de las tecnologías habilitadoras [2] (aquéllas que aseguran su correcta operación y permiten el despliegue de aplicaciones elementales y servicios sectoriales). Sin embargo, el trabajo relacionado con la adaptabilidad y las capacidades cognitivas de IoE donde "todas las cosas" deberían mejorar su disposición para aprender, comprender y actuar en el mundo físico, el digital y el social no ha hecho más que comenzar. Desde las TIC, entendemos que el aumento de la inteligencia en IoE debe interpretarse como la capacidad de los sistemas que la componen de aprender de su experiencia y en base a ésta adaptar/mejorar sus prestaciones [3], permitiendo la asignación inteligente de recursos, la operación automática de red y la provisión inteligente de servicios.

La investigación y el desarrollo hacia la IoE inteligente es un empeño ambicioso y altamente interdisciplinar que debe abordarse en los diferentes niveles de su arquitectura. Se han publicado numerosos trabajos describiendo arquitecturas IoE multicapa [4-6]. 
A grandes rasgos, existen tres niveles que interactúan: (i) la red de comunicación (y especialmente su tecnología de subred de acceso), (ii) la nube (cloud), y (iii) la capa de aplicación. En este contexto, el principal objetivo del proyecto AIM es contribuir a la extensión, inteligencia, adaptabilidad cognitiva, eficiencia y aporte de valor del paradigma de IoE, explorando soluciones TIC factibles en cada uno de los niveles citados. De entre los diferentes bloques de trabajo que componen este proyecto, nos centraremos en este artículo en la capa de aplicación de IoE y en el papel que la calidad de experiencia de usuario (Quality of user Experience, QoE) puede tener en este entorno.

El resto del artículo se organiza como se indica a continuación. La sección II describe la capa de aplicación en IoE y cómo esta se convierte en un elemento que proporciona valor añadido y donde la QoE, en términos de cómo alcanzarla y en cómo medirla, se convierte en un reto a resolver. La sección III explora los modelos de evaluación de la QoE para IoE presentados en la literatura especializada. Finalmente, el artículo finalizada en la sección IV, donde se discute la situación actual en base a la información revisada y se propone el trabajo futuro a realizar.

\section{LA CAPA DE APLICACIÓN EN IOE}

Nos centramos en este apartado en describir la creación de valor añadido en la capa de aplicación de IoE, más allá de las expectativas estándar, aportando ventajas competitivas. El aumento de la inteligencia, de la eficiencia y del número de dispositivos conectados en IoE proporcionará al sector productivo no sólo mejores prestaciones sino también la posibilidad de explotar la información fruto de los propios procesos, por lo que la creación de valor será un elemento más de la cadena de suministro [7]. Aquí surgen nuevas cuestiones: ¿cómo diseñar y llevar a cabo la necesaria provisión de recursos para implementar un servicio/aplicación IoE? ¿Cómo evaluar las prestaciones de estos servicios/aplicaciones para satisfacer niveles apropiados de calidad de servicio (Quality of Service, QoS) o de experiencia (Quality of user Experience, QoE)? ¿Qué implica para una compañía/usuario final una aplicación cognitiva en IoE? ¿Cómo se puede medir la inteligencia alcanzable por el despliegue de tales servicios/aplicaciones? Etc. Las respuestas pasan por evaluar las prestaciones de IoE, lo que no es tarea sencilla. IoE proporciona un entorno de creación de servicios dinámico para nuevas aplicaciones y medios y herramientas para monitorizar los procesos de negocio o los parámetros de red, pero a la vez, está sujeta a restricciones comunes como la energética, la adaptación en tiempo real a la adquisición de datos o la tolerancia a fallos. Si bien los modelos de evaluación de prestaciones para redes tradicionales han sido ampliamente estudiados, para IoE están todavía en sus inicios [8].

Algunos autores coinciden en la necesidad de tener en cuenta nuevos factores en la evaluación de prestaciones de IoE, y en la definición de nuevas métricas para capturar el comportamiento tanto de capas individuales como de la arquitectura completa. Existen experiencias preliminares del Grupo de investigación de Ingeniería Telemática de la Universidad Politécnica de Cartagena al respecto [9]. Como evolución natural de trabajos de investigación previos, proponemos seguir las indicaciones de uno de los pocos artículos con esta visión sobre el nuevo paradigma cognitivo IoE [10]. En él, se dividen las métricas en dos dimensiones, coste y beneficio. La dimensión beneficio se refiere a métricas tales como calidad de los datos, de la información y de experiencia. La primera pretende evaluar la calidad de los datos adquiridos a nivel de dispositivo y hace referencia por ejemplo a su exactitud, veracidad, integridad o actualidad. La calidad de la información está íntimamente ligada a la información a nivel de toma de decisiones, una vez la comunican y procesan el dispositivo, las redes y la nube. Por último, la QoE se contempla como "the overall acceptability of an application or service, as perceived subjectively by the end user", según se define por la International Telecommunications Union (ITU) [11]. Esta métrica se puede extender más allá de la clásica provisión de QoS, teniendo en cuenta por ejemplo, el acceso a la IoE de dispositivos móviles o estáticos mediante una conexión apropiada, la capacidad de la comunicación para garantizar la ejecución de una aplicación, la disponibilidad de suficientes recursos de computación a nivel del cloud o el efecto del servicio que percibe el usuario final. Por otro lado, la dimensión coste permite estimar la eficiencia de la utilización de los recursos. La eficiencia de recursos incluye figuras de mérito relacionadas con la eficiencia en la utilización de múltiples dispositivos o individual, la carga computacional en que se incurre en escenarios multiaplicación, el consumo energético en los distintos niveles de la arquitectura, la eficiencia de los recursos de almacenamiento, etc. Los trabajos de investigación actuales están todavía lejos de una visión de IoE cognitiva. La definición, la caracterización y la evaluación de estas métricas de evaluación de prestaciones contribuirán a conocer mejor los sistemas IoE a gran escala y a cerrar la brecha actual entre teoría $\mathrm{y}$ práctica para dimensionar y planificar a nivel de sistema los servicios y aplicaciones.

\section{IDENTIFICACIÓN DE MÉTRICAS}

Como se ha indicado anteriormente, $\mathrm{Wu}$ propone en [10] una separación de los componentes que determinan las prestaciones en términos de calidad para la IoE en tres capas: calidad de datos (Quality of Data, QoD), calidad de la información (Quality of Información, QoI) y calidad de experiencia de usuario (Quality of user Experience, QoE) como se muestra en la Tabla I. Esta misma clasificación de prestaciones en tres niveles, entiéndase QoD, QoI y QoE, también se emplea en [12]. En este trabajo, los autores intentan fusionar los conceptos de Social Internet of Things 
(SIoT) y Cognitive Internet of Things (CIoT) dentro del paradigma de IoE, presentando un ejemplo de implementación con smart software agents.

Para la QoD, Wu [10] se basa en el trabajo anterior realizado por Bellavista et al. [13], donde se propone medir la calidad de los datos recolectados, ya sea a través de la captura mediante sensores $u$ otros componentes/servicios, y su impacto en el contexto. Respecto a QoI, su definición la extrae de [14], donde se introduce un modelo paramétrico de medida dado por (1), donde las componentes están normalizadas. Claramente podemos observar que hay métricas duplicadas en los niveles QoD y QoI, por ejemplo la precisión. Según [10], la diferencia radica en la unidad lógica que se está midiendo, siendo en QoD el dato en sí mientras que en QoI es la información, con un nivel más alto de procesado. No obstante, este (posible) solape puede ser contradictorio como discutiremos más adelante.

Por último, se incluye en la taxonomía la capa QoE. En ella se diferencian cuatro subniveles (véase la Tabla I). El subnivel comunicación entendemos que se correspondería con las métricas clásicas de QoS, esto es, pérdidas de paquetes, retardo, jitter y ancho de banda. También podrían añadirse aquí otras métricas empleadas en modelos paramétricos de QoE obtenidos a partir de mediciones objetivas de un servicio, como por el ejemplo el tiempo de buffering, el codec empleado en una transmisión de video o la tasa de codificación de bits. Si además juntamos este subnivel de comunicación con el subnivel acceso, entendemos que nos encontramos aquí con los modelos de medición de QoE propuestos en la literatura especializada, como pueden ser el modelo E (E-model, [15]) para Voz sobre IP (VoIP), que específicamente incluye un parámetro denominado Access y que hace referencia al tipo de acceso al servicio que tiene el usuario, o los modelos ITU-T P.1201 [16] e ITU-T P.1202 [17] para la medición de QoE en servicios de transmisión de video. El subnivel aplicación intentaría capturar aquellos parámetros subjetivos propios del consumo de un servicio, y cuyo impacto en la QoE ha quedado demostrado en trabajos previos, como son la interfaz gráfica o la facilidad de uso [18] [19].

$$
Q o I=Q \cdot P \cdot R \cdot A \cdot D \cdot T \cdot V
$$

Un aspecto diferenciador de la propuesta de evaluación de prestaciones de calidad de [10] es la introducción de un elemento "negativo", el coste, al que hemos denominado Quality Cost (QC), a semejanza de los modelos de análisis coste-beneficio muy empleados en teoría de decisión en disciplinas como la economía o la biología, entre otras. Aunque sí que existen algunos trabajos en la literatura especializada que abordan la compensación entre QoS y eficiencia energética [2022], no se había planteado desde una perspectiva más amplia para QoE y con otros factores reductores de la calidad. Si miramos en detalle la Tabla I, el efecto de la eficiencia computacional y del almacenamiento de información se verá sin duda minimizado por la integración y colaboración entre la IoE y el cloud. No obstante, el uso o la delegación de estas tareas al cloud

Tabla I

DESCRIPCIÓN DE LA PROPUESTA DE MÉTRICAS DE EVALUACIÓN DE PRESTACIONES INTRODUCIDA EN [10]

\begin{tabular}{|c|c|c|c|c|}
\hline Capa o Nivel & Métrica & Descripción & Beneficio & Coste \\
\hline \multirow{4}{*}{$\begin{array}{l}\text { Quality of Data } \\
\qquad \text { (QoD) }\end{array}$} & Data accuracy & Precisión & $\mathrm{X}$ & \\
\hline & Data truthfulness & Grado de fiabilidad & $\mathrm{X}$ & \\
\hline & Data completeness & Ratio de datos adquiridos sobre requeridos & $\mathrm{X}$ & \\
\hline & Data up-to-dateness & Validez en la toma de decisión & $\mathrm{X}$ & \\
\hline \multirow{7}{*}{$\begin{array}{l}\text { Quality of } \\
\text { Information } \\
\text { (QoI) }\end{array}$} & Quantity $(Q)$ & Cuánta información válida se ha obtenido para la toma de decisión & $\mathrm{X}$ & \\
\hline & Precission $(P)$ & $\begin{array}{l}\text { Proporción de información relevante respecto a toda la información } \\
\text { capturada por sensores/redes/servicios }\end{array}$ & $\mathrm{X}$ & \\
\hline & Recall (R) & $\begin{array}{l}\text { Proporción de información relevante respecto a toda la información } \\
\text { capturada sin sensores/redes/servicios }\end{array}$ & $\mathrm{X}$ & \\
\hline & Accuracy $(A)$ & Grado de precisión según los requisitos del tomador de decisiones & $\mathrm{X}$ & \\
\hline & Detail (D) & Grado de completación de cara al tomador de decisiones & $\mathrm{X}$ & \\
\hline & Timeliness $(T)$ & $\begin{array}{l}\text { Relación entre la información obtenida y el momento de uso para la } \\
\text { toma de decisión (inversamente proporcional al retardo) }\end{array}$ & $\mathrm{X}$ & \\
\hline & Validity $(V)$ & Veracidad de la información obtenida & $\mathrm{X}$ & \\
\hline \multirow{4}{*}{$\begin{array}{l}\text { Quality of user } \\
\text { Experience } \\
\text { (QoE) }\end{array}$} & Access & Conexión & $\mathrm{X}$ & \\
\hline & Communication & Mediciones de la calidad de la comunicación, QoS & $\mathrm{X}$ & \\
\hline & Resources & $\begin{array}{l}\text { Disponibilidad de recursos, por ejemplo para servicios con altas } \\
\text { demandas de computación }\end{array}$ & $\mathrm{X}$ & \\
\hline & Application & $\begin{array}{l}\text { Variables que afectan directamente al usuario (p.e., facilidad de } \\
\text { uso) }\end{array}$ & $\mathrm{X}$ & \\
\hline \multirow{4}{*}{$\begin{array}{l}\text { Quality Cost } \\
\text { (QC) }\end{array}$} & $\begin{array}{l}\text { Device utilization } \\
\text { efficiency }\end{array}$ & Grado eficiencia en la utilización de los recursos & & $\mathrm{X}$ \\
\hline & $\begin{array}{l}\text { Computation } \\
\text { efficiency }\end{array}$ & $\begin{array}{l}\text { Grado de eficiencia computacional (recursos de cómputo en la } \\
\text { CIoT) }\end{array}$ & & $\mathrm{X}$ \\
\hline & Energy efficiency & $\begin{array}{l}\text { Grado de eficiencia energética de los dispositivos en toda su } \\
\text { perspectiva: cómputo, acceso a red, comunicación, etc. }\end{array}$ & & $\mathrm{X}$ \\
\hline & Storage efficiency & $\begin{array}{l}\text { Grado de habilidad para el almacenamiento físico (y su gestión) de } \\
\text { la información }\end{array}$ & & $\mathrm{X}$ \\
\hline
\end{tabular}


podría tener un impacto en otras métricas, como por ejemplo un aumento en el gasto energético derivado de una mayor necesidad de comunicación con la red (en particular con la nube) o por ejemplo una mayor probabilidad de error en el envío de los datos (impactando entonces en la QoS).

Otra propuesta de evaluación de calidad en IoE se presentó en [23]. Los autores proponen dividir la QoE en diferentes dominios, asociados a los diferentes niveles de una arquitectura IoT/IoE, y medir la QoE como la combinación de la calidad en cada dominio individual. Los niveles empleados son cinco: dispositivos físicos, red, combinación (en referencia a la virtualización y la integración con la nube), aplicación y contexto. La Tabla II resume la descripción de cada uno de ellos. Los autores evalúan de forma práctica su modelo con un despliegue experimental que también incluye mediciones subjetivas de calidad para comparación. Aunque obtienen un modelo simple de medición de QoE, éste es muy limitado en cuanto a variables (métricas) empleadas. Como principal resultado destacan la baja influencia de la calidad de video sobre la QoE final, aunque no queda claro en qué capa de su propuesta se ubicaría (physical layer o network layer). Desde un punto de vista más social, algunos investigadores [24] proponen asociar directamente la QoE en IoE a Net Promoter Score (NPS) [25]. NPS es un indicador de la lealtad de los clientes a un producto basado en una única pregunta que responden los clientes: "con qué probabilidad recomendarías este producto o servicio a un familiar o amigo?". Esta propuesta, aunque interesante, presenta bastantes controversias, la principal su excesiva simplicidad.

En [26], los autores sugieren un modelo para la medición de la QoE en IoT también basado en niveles, en este caso tres: usuario, servicio y contexto (véase la Tabla III). La explicación de cada una de estas tres componentes es muy limitada y aunque los autores llevan a cabo un análisis posterior, para éste sólo emplean como métricas el tiempo de respuesta, la tasa de transmisión, la estabilidad y la precisión, limitando su aplicabilidad.

Por último, hacemos referencia a un interesante trabajo realizado en [27]. En este caso, el modelado de QoE se hace respecto a cuatro capas: dispositivos, red, computing, e interfaz de usuario (véase Tabla IV). Como novedad adicional, se definen dos tipos de métricas: métricas físicas y métricas metafísicas. Para cada capa se definen un conjunto (limitado) de métricas físicas, como por ejemplo resolución de cámaras (capa device), ancho de banda (capa network), prestaciones del servidor en la nube (capa computing) o presentación de la aplicación (capa user interface). Respecto a las métricas metafísicas, éstas intentan capturar la característica cognitiva de la IoT, y para ello los autores proponen relacionarlas con el logro de un objetivo. En concreto, como propuesta inicial, las asocian a obtener información del contexto o a disponer de un servicio cómodo. Una vez definidas e identificadas las métricas metafísicas (p.e. precisión, cantidad, timeliness, etc.) los autores modelan la relación entre métricas (físicas y metafísicas) y a continuación, modelan la relación entre las métricas metafísicas y la QoE de la aplicación. No obstante, los autores dejan justamente el modelado como trabajo futuro a desarrollar.

Tabla II

MÉTRICAS IDENTIFICADAS PARA LA EVALUACIÓN DE QOE EN IOT/IOE SEGÚN [23]

\begin{tabular}{clc}
\hline $\begin{array}{c}\text { Dominio o } \\
\text { nivel }\end{array}$ & \multicolumn{1}{c}{ Descripción } & $\begin{array}{c}\text { Asociación con } \\
\text { modelo [10] de } \\
\text { Tabla I }\end{array}$ \\
\hline $\begin{array}{c}\text { Physical } \\
\text { devices }\end{array}$ & $\begin{array}{l}\text { Medición de la calidad que } \\
\text { proporcionan los elementos } \\
\text { físicos de la IoE }\end{array}$ & $\begin{array}{c}\text { QoD, QoE } \\
\text { (QoS) }\end{array}$ \\
\hline Network layer & $\begin{array}{l}\text { Prestaciones de la } \\
\text { infraestructura de red sobre la } \\
\text { que opera la IoE }\end{array}$ & QoE (QoS) \\
\hline Combination & $\begin{array}{l}\text { Medición de la interacción con } \\
\text { el cloud. Objetos virtuales y su } \\
\text { combinación interaccionando } \\
\text { con objetos físicos }\end{array}$ & QoI, QoE \\
\hline Application & $\begin{array}{l}\text { Calidad en términos de control, } \\
\text { interactividad, presentación y } \\
\text { usabilidad }\end{array}$ & QoE \\
\hline Context layer & $\begin{array}{l}\text { Efecto del contexto de uso, } \\
\text { p.e., tipo de dispositivo, tipo de } \\
\text { consumidor, precio, ubicación } \\
\text { geográfica,... }\end{array}$ & QoE \\
\hline \hline
\end{tabular}

Tabla III

MÉTRICAS IDENTIFICADAS PARA LA EVALUACIÓN DE QOE EN IOT/IOE SEGÚN [26]

\begin{tabular}{clc}
\hline \hline $\begin{array}{c}\text { Dominio } \\
\text { o nivel }\end{array}$ & \multicolumn{1}{c}{ Descripción } & $\begin{array}{c}\text { Asociación con } \\
\text { modelo [10] de } \\
\text { Tabla I }\end{array}$ \\
\hline User & $\begin{array}{l}\text { Métricas: background, experience, } \\
\text { expectation, physiological and } \\
\text { mental state }\end{array}$ & QoE \\
\hline \multirow{5}{*}{ Service } & $\begin{array}{l}\text { Métricas: Transmission layer (p.e., } \\
\text { métricas de red), application layer } \\
\text { (p.e., conversión, resolución de } \\
\text { video, etc.), service layer } \\
\text { (prioridades) }\end{array}$ & QoE, QoI \\
\hline \multirow{5}{*}{$\begin{array}{l}\text { Context } \\
\text { Physical context (condiciones } \\
\text { hardware and software) y external } \\
\text { context (entorno social, cultural, } \\
\text { etc.) }\end{array}$} & QoE \\
\hline \hline
\end{tabular}


Tabla IV

MÉTRICAS IDENTIFICADAS PARA LA EVALUACIÓN DE QOE EN IOT/IOE SEGÚN [27]

\begin{tabular}{clc}
\hline \hline Capa o nivel & \multicolumn{1}{c}{ Descripción } & $\begin{array}{c}\text { Asociación con } \\
\text { modelo [10] de } \\
\text { Tabla I }\end{array}$ \\
\hline Device & $\begin{array}{l}\text { Calidad de la generación de datos } \\
\text { brutos }\end{array}$ & QoD \\
\hline Network & $\begin{array}{l}\text { Calidad en la transferencia de } \\
\text { datos }\end{array}$ & QoE (QoS) \\
\hline Computing & $\begin{array}{l}\text { Calidad en el procesado y } \\
\text { análisis de los datos } \\
\text { (almacenamiento, abstracción, } \\
\text { indexación, prestaciones CPU, } \\
\text { precisión,...) }\end{array}$ & QoI, QoE \\
\hline $\begin{array}{c}\text { User } \\
\text { interface }\end{array}$ & $\begin{array}{l}\text { Calidad en la interacción } \\
\text { aplicación-usuario }\end{array}$ & QoE \\
\hline \hline
\end{tabular}

\section{DISCUSIÓN Y CONCLUSIONES}

Como se indica en todos los trabajos referenciados, aún supone un reto el estudio y análisis de la QoE a nivel de sistema, de forma generalizada. Tras describir las diferentes visiones holísticas propuestas en la literatura, surgen algunas inquietudes. En primer lugar creemos que existe una clara duplicación de métricas. Será necesario determinar si realmente es necesario incluirlas en diferentes niveles o si su impacto puede absorberse en un único nivel. En segundo lugar, es necesario un estudio detallado de qué métricas han de tenerse en cuenta. Ya sean métricas físicas con una relación directa en la QoE, como se indicaba en [27], o una combinación de éstas donde se podría emplear una metodología similar a los árboles de decisión, aunando las sugerencias realizadas en [23] y [27]. Por último, la tendencia de dar respuesta a la QoE como la unión del nivel de calidad en varios niveles o capas parece acertada. No obstante, la relación (el modelado) entre los distintos niveles no ha sido definido en ninguno de los trabajos considerados. Además, creemos necesario profundizar en el estudio de la inclusión de un componente "negativo" en la formulación final de la QoE, como podría ser el Quality Cost.

Consideramos por tanto necesario un nuevo enfoque para la evaluación de prestaciones de los servicios y aplicaciones de la IoE capaz de capturar su idiosincrasia, es decir, las nuevas métricas que definen calidad, nivel de conocimiento, nivel de inteligencia, consumo, etc. En consecuencia, uno de los objetivos del proyecto AIM será proporcionar una guía rigurosa para la evaluación de prestaciones, la planificación y el dimensionado de los servicios y aplicaciones en IoE. Para ello, planteamos dos fases. Una primera fase en la que definir, caracterizar y cuantificar nuevas métricas que en base a una visión holística satisfagan QoE en IoE y una segunda fase de búsqueda de diseño de herramientas de optimización capaz de capturar la relación entre los distintos niveles en los que se categorice la QoE.

\section{AGRADECIMIENTOS}

This research was supported by the AEI/FEDER, UE project grant TEC2016-76465-C2-1-R (AIM).

\section{REFERENCIAS}

[1] I. Bojanova, G. Hurlburt, J. Voas, "Imaginering an Internet of Anything”, Computer, Vol. 47 (6), pp. 72-77, 2014.

[2] A. Al-Fuqaha, M. Guizani, M. Mohammadi, M. Aledhari, M. Ayyash, "Internet of Things: A Survey on Enabling Technologies, Protocols, and Applications", IEEE Communications Surveys \& Tutorials, Vol. 17 (4), pp. 2347$2376,2015$.

[3] A. Sheth, "Internet of Things to Smart IoT through Semantic, Cognitive, and Perceptual Computing", IEEE Intelligent Systems, Vol. 31 (2), pp. 108-112, 2016.

[4] J. Lloret, J. Tomas, A. Canovas, L. Parra "An Integrated IoT Architecture for Smart Metering", IEEE Communications Magazine, Vol. 54 (2), pp.50-57, 2016.

[5] S. M. A. Oteafy, H. S. Hassanein, "Resilient IoT Architectures Over Dynamic Sensor Networks With Adaptive Components", IEEE Internet of Things Journal, vol. 4 (2), ,pp. 474-483, 2017.

[6] N. Kaur, S. K. Sood, "An Energy-Efficient Architecture for the Internet of Things (IoT)", IEEE Systems Journal, Vol. PP (99), pp. 1-10, 2015.

[7] M. Raynor, M. Cotteleer, "The more things change: Value creation, value caption, and the Internet of Things", Deloitte Univ. Press, 2015. [Online] http://goo.gl/XJK09n/

[8] A. Floris et al., "Quality of Experience in the Multimedia Internet of Things: definition and practical use-cases", in Proc. ICC15, pp. 1747-1752, 2015.

[9] R. Sanchez-Iborra, M.-D. Cano, "JOKER: A Novel Opportunistic Routing Protocol", IEEE J. of Selected Areas in Communications, 2016. DOI: 10.1109/JSAC.2016.2545439

[10] Q. Wu et al., "Cognitive Internet of Things: A New Paradigm Beyond Connection", IEEE Internet of Things Journal 1(2), 2014.

[11] "ITU-T: New definitions for inclusion in Recommendation ITUT P.10/G.100", ITU-T Recommendation P.10/G.100 Amend. 2, 2008.

[12] P. Kasnesis, C. Z. Patriakekios, D. Kogias, L. Toumanidis, I.A. Venieris, "Cognitive friendship and goal management for the social IoT", Computers and Electrical Engineering, Vol. 58, pp. 412-428, 2017.

[13] P. Bellavista, "A survey of context data distribution for mobile ubiquitous systems", ACM Computing Surveys (CSUR), vol. 44 (4), pp. 1-49, 2012.

[14] J. Mitola, "Cognitive radio architecture evolution", Proceedings of IEEE, vol. 97 (4), pp. 626-641, 2009.

[15] "The E-model, a computational model for use in transmission planning", ITU-T Rec. G.107, 2005.

[16] "ITU-T: Parametric non-intrusive assessment of audiovisual media streaming quality", ITU-T Recomm. P.1201, 2012.

[17] "ITU-T: Parametric non-intrusive bitstream assessment of video media streaming quality", ITU-T Recomm. P.1202, 2012.

[18] Maria-Dolores Cano, F. Cerdan, S. Almagro, "Statistical Analysis of a Subjective QoE Assessment for VVoIP Applications", ETRI Journal Vol. 32 (6), pp. 2010.

[19] Dong-Hee Shin, "Conceptualizing and measuring quality of experience of the Internet of Things: Exploring how quality is perceived by users", Information \& Management, in press, pp. 1$14,2017$.

[20] Zaheeruddin, D.K. Lobiyal, Sunita Prasad, "Ant based Pareto optimal solution for QoS aware energy efficient multicast in wireless networks", Applied Soft computing, Vol. 55, pp. 72-81, 2017.

[21] Qiaoni Han, Bo Yang, Cailian Chen, Xinping Guan, "Energyaware and QoS-aware load balancing for HetNets powered by renewable energy", Computer Networks, Vol. 94 (15), pp. 250$262,2016$.

[22] Chi Harold Liu, Jun Fan, Joel W. Branch, Kin K. Leung, "Toward QoI and Energy-Efficiency in Internet-of-Things Sensory Environments", IEEE Transactions on Emerging Topics in Computing, Vol. 2 (4), pp. 473-487, 2014. 
[23] A. Floris, L. Atzori, "Quality of Experience in the Multimedia Internet of Things: definition and practical use-cases", in Proc. International Conference on Communicaitons (ICC) - Workshop on Quality of Experience-based Management for Future Internet Applications and Services (QoE-FI), pp. 1747-1752, 2015.

[24] M. Aazam, M. St-Hilaire, Chung-Horng Lung, I. Lambadaris "MeFoRE: QoE based resource estimation at Fog to enhance QoS in IoT", in Proc. 23rd International Conference on Telecommunications, pp. 1-5, 2016.

[25] F. F. Reichheld, "The One Number You Need to Grow", Harvard Business Rewiew, 2003.
[26] L. Li, M. Rong, G. Zhang, "An Internet of Things QoE evaluation method based on multiple linear regression analysis", in Proc. 10th International Conference on Computer Science \& Education (ICCSE), pp. 925-928, 2015.

[27] Y. Ikeda, S. Kouno, A. Shiozu, K. Noritake "A framework of scalable QoE modeling for application explosion in the Internet of Things", in Proc. IEEE 3rd World fórum on Internet of Things (WF-IoT), pp. 425-429, 2016. 